\title{
An alarm pheromone modulates appetitive olfactory learning in the honeybee (Apis mellifera)
}

\author{
Elodie Urlacher, Bernard Francés ${ }^{\dagger}$, Martin Giurfa $^{\dagger}$ and Jean-Marc Devaud ${ }^{*,+}$ \\ Research Center on Animal Cognition, National Center for Scientific Research, University Paul Sabatier, Toulouse, France
}

\section{Edited by:}

Jean-Christophe Sandoz, National

Center for Scientific Research, France

Reviewed by:

Ricarda Scheiner, Technical University

of Berlin, Germany

Judith Reinhard, The University of

Queensland, Australia

*Correspondence:

Jean-Marc Devaud, Research Center on Animal Cognition - National Center for Scientific Research Joint Research Unit 5169, Paul Sabatier University, 118 route de Narbonne, 31062 Toulouse

Cedex 9, France.

e-mail: devaud@cict.fr

${ }^{\dagger}$ Bernard Francés, Martin Giurfa and Jean-Marc Devaud have contributed equally to this work
In honeybees, associative learning is embedded in a social context as bees possess a highly complex social organization in which communication among individuals is mediated by dance behavior informing about food sources, and by a high variety of pheromones that maintain the social links between individuals of a hive. Proboscis extension response conditioning is a case of appetitive learning, in which harnessed bees learn to associate odor stimuli with sucrose reward in the laboratory. Despite its recurrent use as a tool for uncovering the behavioral, cellular, and molecular bases underlying associative learning, the question of whether social signals (pheromones) affect appetitive learning has not been addressed in this experimental framework. This situation contrasts with reports underlining that foraging activity of bees is modulated by alarm pheromones released in the presence of a potential danger. Here, we show that appetitive learning is impaired by the sting alarm pheromone (SAP) which, when released by guards, recruits foragers to defend the hive. This effect is mimicked by the main component of SAP, isopentyl acetate, is dose-dependent and lasts up to $24 \mathrm{~h}$. Learning impairment is specific to alarm signal exposure and is independent of the odorant used for conditioning. Our results suggest that learning impairment may be a response to the biological significance of SAP as an alarm signal, which would detract bees from responding to any appetitive stimuli in a situation in which such responses would be of secondary importance.

Keywords: honeybee, learning, pheromone, stress, alarm, olfactory, modulation, insect

\section{INTRODUCTION}

Social insects have evolved sophisticated communication systems, which include behavioral displays such as the honeybee dances (Frisch, 1967) and chemical signals that play a crucial role in the coordination of individual behaviors inside a colony. Bees, ants, and wasps release a high variety of chemical compounds that act as pheromones, thus ensuring intraspecific chemical communication and adaptive responses to a variety of stimuli across different timescales (Wilson, 1971; Vander Meer et al., 1997; Wilson and Hölldobler, 2009). While primer pheromones induce long-lasting changes in physiology and behavior, releaser pheromones trigger rapid and short-term behavioral responses (Wilson and Hölldobler, 2009).

Honeybee pheromones have been the subject of intensive studies (Free, 1987) which have focused on their multiple behavioral and physiological consequences. Among the pheromones released by worker bees, the sting alarm pheromone (SAP), a releaser pheromone contained in the sting chamber, prompts stinging and fast recruiting of nest-mates to defend the resources of the colony when released by guards facing a potential danger (Free, 1987). Several studies have shown that SAP acts as a modulator of the sensitivity to environmental stimuli, as assessed by the quantification of reflex responses. In particular, exposure to some of its main components changes the responsiveness (as usually measured by the threshold value of a given stimulus eliciting a response) to appetitive or nociceptive stimuli: it decreases responsiveness to sucrose (Balderrama et al., 2002) and, depending on the nature and dose of the SAP compound, increases or decreases responsiveness to electric shocks (Núñez et al., 1998; Balderrama et al., 2002).
It has been proposed that response thresholds are a key parameter of social regulation in insects (Robinson and Page, 1989; Rueppell et al., 2006). For example, the probability to forage for pollen outside the hive is linked to increased responsiveness to sucrose, and possibly to light (Page and Erber, 2002; Pankiw, 2003, 2005; Ben-Shahar, 2005; Page et al., 2006). Since pheromones modulate sensory response thresholds, they can also affect the probability of performing certain behaviors. Moreover, pheromones can also affect plastic behaviors such as learning, as shown recently by studies on queen mandibular pheromone (QMP) (Vergoz et al., 2007a). This pheromone blocks aversive associative learning in young bees (Vergoz et al., 2007a), in addition to triggering reflex responses such as feeding and grooming the queen (Free, 1987). However, QMP does not affect appetitive learning in young bees, thus specifically preventing aversive experiences that young bees could have in the vicinity of their queen.

Appetitive learning is particularly important during foraging, as it requires to associate floral aromas with the presence of pollen or nectar (Giurfa, 2007). SAP can rapidly induce bees to quit foraging and to reduce recruitment of other bees for foraging by producing a vibrational "stop signal" (Nieh, 2010). Hence, we wondered whether this effect might be accompanied by a change in appetitive learning, which drives foraging activities (Menzel, 1985; Giurfa, 2007). Appetitive learning can be easily studied in controlled conditions using proboscis extension response (PER) conditioning (Takeda, 1961; Bitterman et al., 1983). In this Pavlovian task, restrained bees are trained to associate an odorant (conditioned stimulus, CS) with a sucrose reward (unconditioned stimulus, US). After 3-5 
trials consisting in paired odorant-sugar presentations, most bees display a conditioned PER to the odorant, which indicates that the association was learnt. Based on its inhibitory effect on foraging activities, we hypothesized that SAP would impair olfactory appetitive learning in the laboratory. Our results show that exposure to SAP or to its main component isopentyl acetate (IPA) does indeed impair appetitive learning, that this effect is SAP-specific and is independent of the odorant used for conditioning.

\section{MATERIALS AND METHODS ANIMALS}

Honeybees from the strain Apis mellifera ligustica were caught at the hive in the morning of each experimental day, cold-anesthetized and restrained in individual harnesses that allowed free movements of the mouthparts and antennae (Bitterman et al., 1983). They were then fed with $5 \mu \mathrm{L}$ of $50 \% \mathrm{w} / \mathrm{w}$ sucrose solution and maintained $2 \mathrm{~h}$ in a dark and humid place. Experiments were performed across several seasons, and thus include winter as well as summer (mostly foraging) bees, of unmatched ages and taken from different hives. These differences may explain the observed variability in learning rates in controls across days and experiments. However, they are unlikely to account for differences between groups as bees from the same hive were assigned to control and experimental groups on every experimental day.

\section{EXPOSURE PROCEDURES}

Two hours after feeding, animals were exposed either collectively to control compounds or to the natural alarm pheromone, or individually to IPA (the main component of SAP) or to solvent. In all cases, exposure lasted $30 \mathrm{~min}$ and was followed by a $30-\mathrm{min}$ rest (unless stated otherwise) before conditioning experiments.

Exposure to the natural pheromone was performed by placing the restrained bees to be conditioned and 50 unrestrained bees in two compartments of a cage $(11 \mathrm{~cm} \times 12 \mathrm{~cm} \times 8.5 \mathrm{~cm})$, separated by a perforated wall allowing odorant diffusion (Figure 1). Electric shocks were delivered to the unrestrained bees through an electric grid connected to a generator whenever they touched the floor of the cage. The bees that received the electric shocks reacted by emitting SAP, which was acknowledgeable to the experimenter by the characteristic posture of the emitting bees (Figure 1; left compartment) and by the typical banana smell of the pheromone (Free, 1987; Núñez et al., 1998). The voltage was increased progressively from $6.5 \mathrm{~V}$ to $9.5 \mathrm{~V}$ during the 30 -min period in order to avoid desensitization. The restrained bees were placed in the other compartment in front of an air extractor (exposed group), so that the pheromone released by the unrestrained bees was blown toward them (Figure 1; right compartment). As a control, another group of unrestrained bees was placed in the setup with the unrestrained bees in a separate compartment, but no shock was delivered to the unrestrained bees (sham). A third control group included bees that were harnessed but not placed in the setup (untreated).

A more controlled protocol was used, in order to avoid possible variations in the amount of SAP received by each individual bee in the cage. It made use of IPA, by adapting previously published procedures (Núñez et al., 1998; Balderrama et al., 2002). IPA, a main component of SAP, can trigger by itself many effects of exposure to the full pheromone blend (Boch et al., 1962; Collins and Blum,

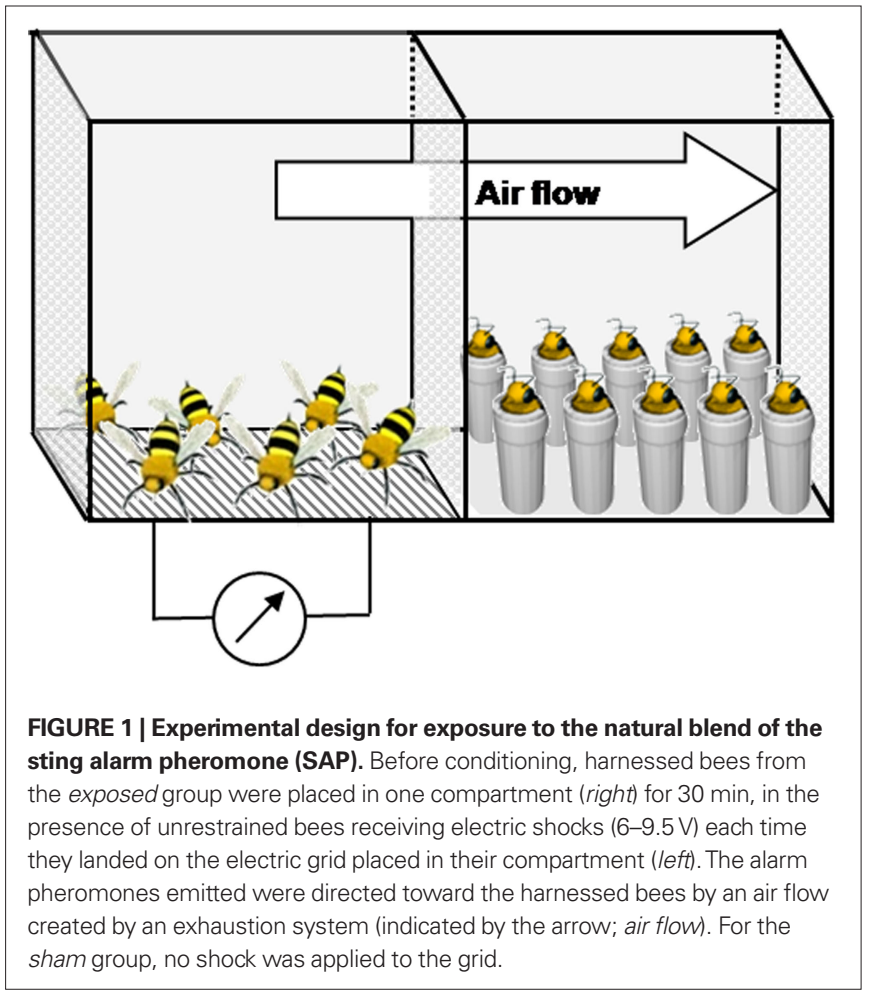

1983). Each restrained bee was placed in an individual $35 \mathrm{~mL}$ glass vial containing a piece of filter paper $(1.5 \times 1.5 \mathrm{~cm})$ soaked with $24 \%$ IPA ( $6 \mu \mathrm{L}$ IPA $+19 \mu \mathrm{L}$ mineral oil), unless specified otherwise. Control bees were handled the same way and exposed to mineral oil alone. In one experiment designed to test for the specificity of the effect of IPA, two additional controls included bees exposed to methyl salicylate or geraniol at the same concentration as IPA.

\section{CONDITIONING}

Bees were subjected to olfactory PER conditioning consisting of three trials, following a standardized protocol described elsewhere in detail (Bitterman et al., 1983). Briefly, each acquisition trial lasted $40 \mathrm{~s}$; it included a familiarization phase of $13 \mathrm{~s}$ in the setup, followed by the forward-paired presentation of an odorant (the CS) and sucrose solution (the US). The presentations of the CS (1-nonanol, pure) and the US (sucrose, 50\% w/w in water) lasted $4 \mathrm{~s}$ and $3 \mathrm{~s}$, respectively, with a $1 \mathrm{~s}$ overlap. Pure odorants are generally used for bee conditioning in order to avoid any concentration decrease due to evaporation over the conditioning trials. In an additional experiment, other odorants (1-hexanol, citral, or nonanal) were used as CSs to make sure that the effect of IPA exposure was not CS-specific. All chemicals were from Sigma-Aldrich (Lyon, France). Before conditioning, all bees were tested for their PER in response to $50 \%$ sucrose. Those bees that failed to respond were discarded; bees that failed to respond to the US during the three conditioning trials were also discarded.

\section{ASSESSMENT OF SENSITIVITY TO SUCROSE}

In order to look for possible effects of IPA exposure on the sucrose responsiveness, unconditioned responses elicited by sucrose were assessed in bees exposed to IPA or mineral oil, using a protocol 
described elsewhere (Scheiner et al., 1999). Those bees had not been conditioned previously, since providing the sucrose reward during conditioning would have modified their responsiveness in the test. Briefly, the bees were first allowed to drink water ad libitum in order to ensure that they would respond specifically to sucrose, and were then presented successively with six sucrose solutions of increasing concentrations $(0.1,0.3,1,3,10$, and $30 \%)$, which were applied on the antennae, interspersed with water stimulations to avoid sensitization. No responses to water were recorded during the experiment; this indicates that the recorded responses to the sucrose solutions were indeed elicited by the sucrose. For each animal, the presence or absence of a PER was recorded for each concentration, and its individual sucrose response score (SRS) was calculated as the number of stimuli eliciting a PER (e.g.) SRS = 3 for an individual responding to 3,10 , and $30 \%$ sucrose but not to lower concentrations). Bees with a SRS of 0 (i.e., not responding to any concentration) were discarded as in the learning experiments (see above).

\section{GENERALIZATION}

In order to test for possible differences in olfactory discrimination, bees were prepared and conditioned as previously but were exposed to IPA, oil, or geraniol after conditioning, instead of before. Exposure started right after the last conditioning trials and lasted $30 \mathrm{~min}$. After a 30 -min rest (i.e., $1 \mathrm{~h}$ post-conditioning), bees were presented with the odorant used as the CS (1-nonanol) and two novel odorants (1-hexanol and nonanal) without reward, with the same timing as during conditioning. These two odorants were chosen given their high (nonanal) and low (1-hexanol) similarity to 1-nonanol (Guerrieri et al., 2005). The order in which the odors were presented was randomized across bees. After the test bees were checked for their PER in response to $50 \%$ sucrose; bees that failed to respond were discarded from the whole experiment.

\section{STATISTICS}

Multiple comparisons were performed using analysis of variance (ANOVA) as the critical conditions required for its application to dichotomous variables were met (at least 40 subjects per group, Lunney, 1970). Although the second criterion (equal sample sizes) was not met, the robustness of the significant effects detected by the ANOVAs was supported by all post hoc analyses (which confirmed all effects), thus suggesting that a requirement for equal group sizes was not critical in our study. Post hoc comparisons (pairwise) were performed using a Chi-square test on the absolute numbers of bees in each category. For the sucrose response analysis, the SRS classes 4-6 were pooled so as to have the required minimum of five individuals per category. In case of multiple comparisons, Bonferroni corrections were applied (wherever applied, the corrected alpha level -0.025 or 0.0125 - is indicated in the text and in the figure legends). All statistics were run on the R software ( $R$ Development Core Team, 2009).

\section{RESULTS}

\section{EXPOSURE TO ALARM PHEROMONES IMPAIRS LEARNING PERFORMANCE}

We studied the effect of exposure to SAP on olfactory PER conditioning, by comparing the learning performances of bees from the SAP-exposed group to those of control bees (sham and untreated groups) (see Materials and Methods). During conditioning, each individual received three paired presentations of 1-nonanol (CS) with sucrose (US) (absolute conditioning). To rule out undesired effects of treatment on sucrose responsiveness and thus on the motivation to learn, all experiments used only bees that responded to the sucrose reward before and after conditioning, and in all three conditioning trials. As shown in Figure 2, bees from the three groups increased their responses to the odor during conditioning trials, thus showing learning of the odor-sucrose association. Accordingly, a two-way repeated-measure ANOVA (Treatment, Trials) revealed that overall bees increasingly responded to the CS across trials (Trial: $F=245.2, \mathrm{df}=2,586, p<0.001$ ). However, learning success differed significantly between groups $(F=3.97$, $\mathrm{df}=2,293, p=0.02$ ), as shown by the proportions of bees displaying a conditioned response to the CS after three conditioning trials. Indeed, SAP-exposed bees exhibited significantly fewer conditioned responses $(52 \%)$ on the last conditioning trial than both control groups. These, in turn, showed very similar performances (untreated: $66 \%$; sham: $68 \% \chi^{2}=0.04, \mathrm{df}=1, p=0.84$ ). This effect of exposure to SAP on learning was significant (controls vs. exposed: $\left.\chi^{2}=5.56, \mathrm{df}=1, p=0.02, \alpha=0.025\right)$.

\section{THE EFFECT OF THE PHEROMONAL BLEND IS MIMICKED BY ITS ACTIVE COMPONENT ISOPENTYL ACETATE (IPA)}

Sting alarm pheromone is a complex blend of about 40 components (Hunt, 2007), among which IPA is sufficient to elicit most of the responses triggered by the entire blend (Boch et al., 1962; Collins and Blum, 1983). Therefore, we tested whether exposure to IPA alone could have similar effects as exposure to the whole pheromone blend. For this, we used a procedure in which individual bees were constantly exposed to a determined amount of IPA, thus ensuring a continuous exposure to a constant amount of odorant.

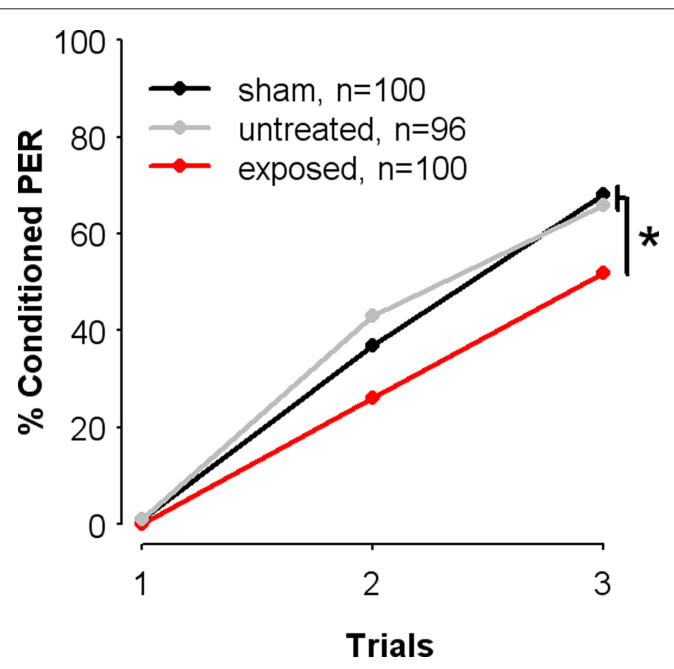

FIGURE 2 | Exposure to the natural blend of the sting alarm pheromone impairs olfactory learning. Learning performances measured as the percentages of conditioned proboscis extension responses (percentage conditioned PER) elicited by the CS, 1-nonanol (conditioned PER) during three conditioning trials. Learning was impaired in bees previously exposed to the SAP (exposed), as compared with control groups (sham and untreated). ${ }^{*} p<0.025$. 
Indeed, in the cage setup used in the previous experiment the SAP concentration could not be controlled precisely since the number of bees releasing it varied over time, and it decreased over the stimulation period as we observed that some of the shocked bees seemed to learn to avoid the grid. We thus asked whether IPA could impair appetitive learning in a similar way as the whole pheromone, and whether this effect depends on the dose of IPA to which the bees are exposed. Different groups of bees were exposed individually to different dilutions of IPA in mineral oil $(4,8,24$, and $40 \%)$ or to oil alone as a control. Figure 3 shows the percentages of conditioned responses obtained in the last trial of the differently exposed groups. Exposure to IPA affected learning in a dose-dependent manner (Dose: $F=4.51, \mathrm{df}=4,237, p=0.0016$ ). While lower concentrations (4 and $8 \%$ ) did not induce a significant response decrease relatively to the control, higher concentrations resulted in lower conditioned responses (Oil/IPA 24\%: $\chi^{2}=6.34, p=0.0117$; Oil/IPA 40\%: $\left.\chi^{2}=10.67, p=0.0011, \alpha=0.0125\right)$. Interestingly, this effect was comparable to that of SAP in the previous experiment, as shown by the similar relative decreases in performances in both conditions (24\% IPA:-23\%; SAP:-19\%). Hence, exposure to a sufficient concentration of IPA allows reproducing the effect of exposure to natural SAP on learning, in a more controlled situation.

In order to test the duration of the effect of the 30-min exposure to IPA, we used the $24 \%$ concentration, which corresponds to the amount of IPA contained in 3-10 stings (Hunt et al., 2003), and for which a clear, significant decrease in conditioned responses was found with respect to the control in the third conditioning trial. We introduced different delays (1.5, 3.5, 5.5, 24, 48, and $72 \mathrm{~h}$ ) between IPA exposure and olfactory conditioning. An independent group was used for each delay. A significant impairment of learning was observed for delays up to $24 \mathrm{~h}$, but not for longer ones (48 and $72 \mathrm{~h}$ ) (Figure 4). Thus, the modulation introduced by IPA induces a long-lasting learning impairment that mimics the effect of exposure to the whole pheromonal blend.
For all further experiments, we used the 24\% IPA dilution, in order to further analyze the modulatory effect of SAP in precisely controlled conditions.

LEARNING IMPAIRMENT IS SPECIFICALLY CAUSED BY EXPOSURE TO IPA Olfactory learning impairment might be the consequence of continuous exposure to any odorant rather than the response to an alarm signal. We thus exposed independent groups of bees to oil,

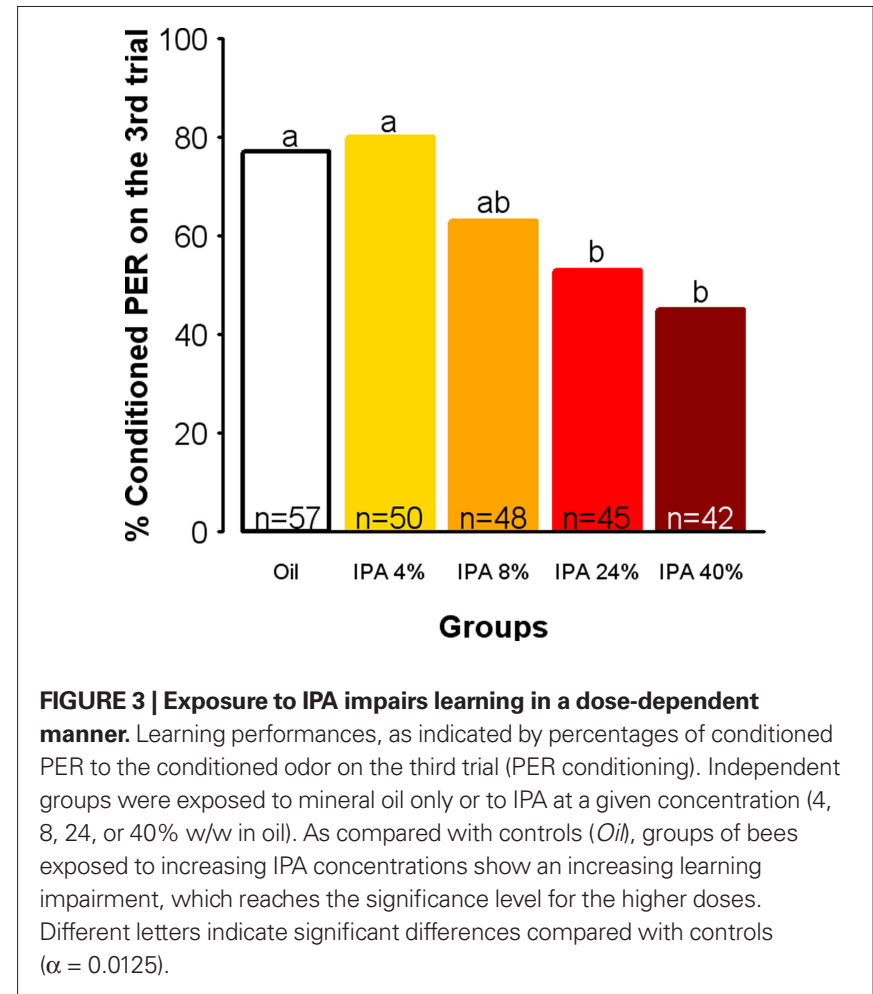

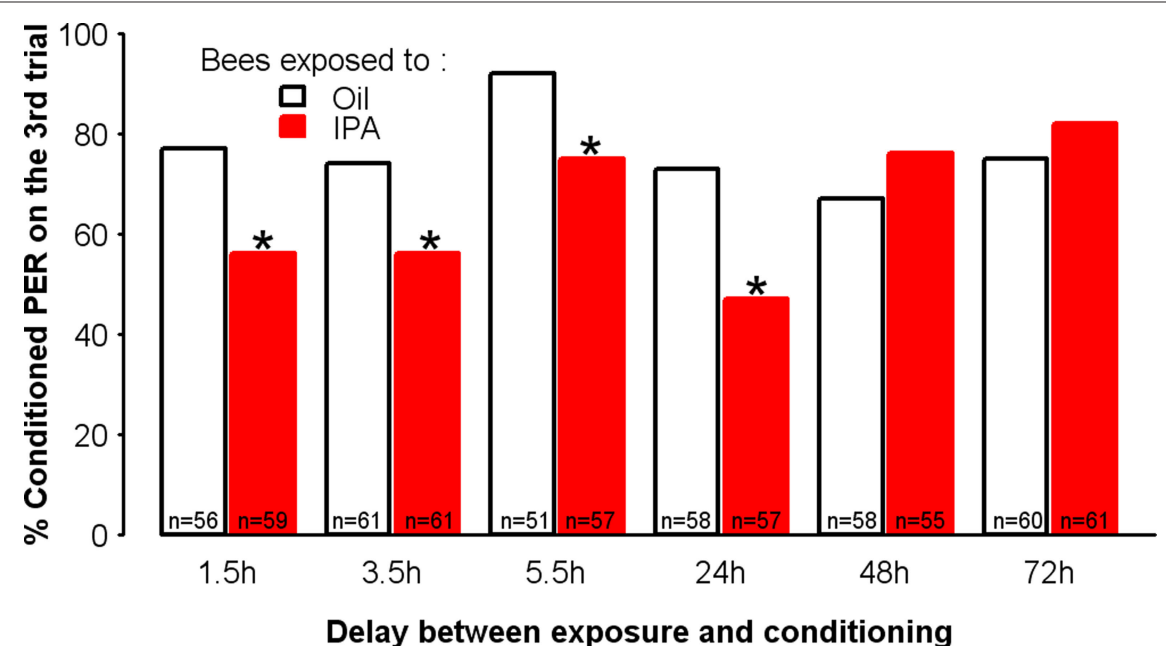

FIGURE 4 | IPA impairs learning up to $\mathbf{2 4} \mathrm{h}$ after exposure. Learning performances, as indicated by percentages of conditioned PER to the conditioned odor on the third trial (PER conditioning), after a delay between exposure (either to IPA at the $24 \%$ concentration or to mineral oil) and olfactory conditioning that varied for each group. Compared with controls (Oil), learning was impaired for delays up to $24 \mathrm{~h}$. By contrast, for delays longer than $24 \mathrm{~h}$, no significant impairment of learning was found. ${ }^{*} p<0.05$ (as compared with respective Oil control groups). 
IPA, methyl salicylate or geraniol (all at the same concentration: $24 \%$ ) for $30 \mathrm{~min}$, and $30 \mathrm{~min}$ after exposure, we conditioned them in parallel, using the same protocol as above (with 1-nonanol as the CS). Methyl salicylate was chosen because it is a non-pheromonal compound with the same functional group (ester) as IPA. Geraniol, on the other hand, is the main component of the attractive Nasonov pheromone used to mark places of interests such as food sources or the hive entrance (Boch and Shearer, 1962; Free, 1987); it offers, therefore, the possibility of testing the effect of another pheromone signal with a different hedonic value from that of the SAP. The effect of geraniol exposure was analyzed relative to that obtained in the two other groups (oil-exposed and IPA-exposed), which were studied in parallel (Figure 5A). Similarly, the effect of methyl salicylate geraniol exposure was compared with that obtained in its corresponding two control groups (oil-exposed and IPA-exposed) (Figure 5B).

Neither methyl salicylate nor geraniol affected learning relatively to the control situation (geraniol/oil: $\chi^{2}=0.75$, $\mathrm{df}=1, p=0.39$; methyl salicylate/oil: $\chi^{2}=0.43, \mathrm{df}=1, p=0.51$ ). Besides, in both cases bees exposed to IPA showed learning performances that were significantly lower than those of bees exposed to methyl salicylate, geraniol, or mineral oil ( $p<0.025$ in all cases). Thus, the decrease in learning induced by IPA was specific to this compound and its alarm message given that geraniol did not induce any decrease in learning. Spurious factors related to olfactory processing such as saturation of odorant receptors as a consequence of exposure can be excluded based on these results.

\section{LEARNING IMPAIRMENT INDUCED BY IPA EXPOSURE IS INDEPENDENT OF THE ODORANT USED AS CONDITIONED STIMULUS}

If exposure to IPA results in a general decrease in appetitive learning, this effect should be observed regardless of the odorant used as the CS. We tested this hypothesis by conditioning independent
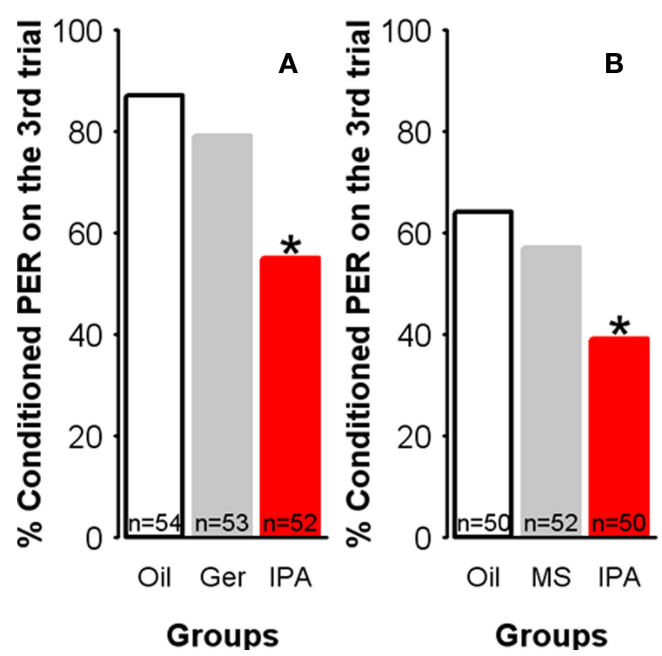

FIGURE 5 | Learning impairment is specific to IPA exposure. Learning performances, as indicated by the percentage of conditioned PER to the conditioned odor on the third trial (PER conditioning), for each group.(A) Compared with controls (Oi), learning remained unaffected after exposure to geraniol (Ger), a compound belonging to the attractive Nasonov pheromone or (B) to methyl salicylate (MS), a non-pheromonal ester. By contrast, bees exposed to IPA show significantly fewer conditioned responses. ${ }^{*} p<0.025$ (as compared with respective control groups). groups of bees using different CSs, after exposure to IPA or to oil for $30 \mathrm{~min}$. As expected, the decrease in learning induced by IPA exposure was independent of the odor used for conditioning. As shown in Figure 6, bees exposed to IPA could learn dissimilar odors such as 1-hexanol, citral, and nonanal but their final level of conditioned responses was always significantly lower than that of control bees exposed to mineral oil (nonanal: $\chi^{2}=4.46, \mathrm{df}=1$, $p=0.035$, citral: $\chi^{2}=4.20, \mathrm{df}=1, p=0.040,1$ hexanol: $\chi^{2}=4.20$, $\mathrm{df}=1, p=0.040)$.

\section{LEARNING IMPAIRMENT DUE TO EXPOSURE TO IPA IS NOT DUE TO A DIMINISHED RESPONSIVENESS TO SUCROSE}

According to previous findings obtained by one of us (Balderrama et al., 2002), we found that exposure to IPA had an impact on responses to sugar. There were significantly more bees failing to display sugar-induced PER at least once during conditioning among those exposed to IPA (23.5\%) than to oil (6.7\%). Such bees were considered to lack the motivation required for optimal learning, and thus were systematically discarded from all experiments. Thus, undesired effects of IPA exposure on sucrose processing could, in principle, be discarded as bees kept in our experiments always responded to the $50 \%$ sucrose solution used as reward. However, IPA might diminish the subjective value of sucrose reward (Scheiner et al., 2004), thus inducing lower conditioning performances in IPAexposed bees. To test this hypothesis, we measured the individual sensitivity to sucrose of both IPA-exposed and control bees, by determining their SRS (see Materials and Methods). This score is a standard measure of sucrose responsiveness in honeybees despite responding identically to the $50 \%$ sucrose solution (Scheiner et al.,

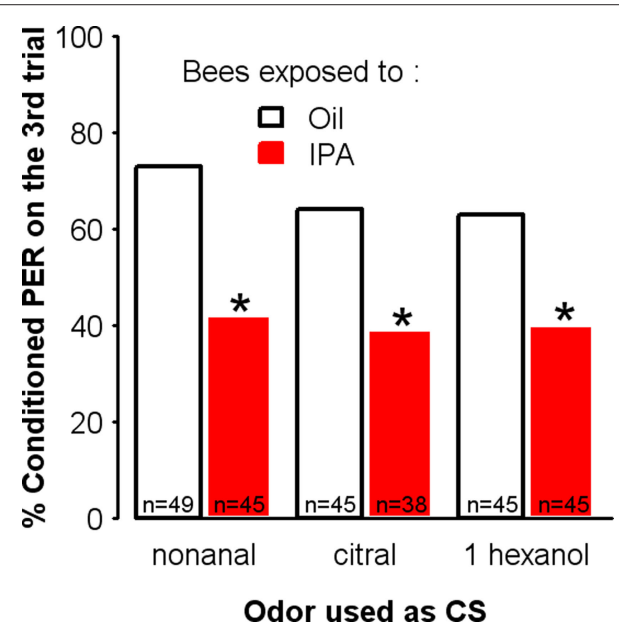

FIGURE 6 | IPA exposure impairs learning irrespective of the odorant used as the conditioned stimulus. Learning performances, as indicated by percentages of conditioned responses to the conditioned odor on the third trial (PER conditioning), for each group. Independent groups of bees were conditioned in a three-trial protocol, after exposure to either IPA $24 \%$ in mineral oil) or mineral oil alone. In each pair of groups, a different odorant (nonanal, citral, or 1-hexanol) was used as the CS during the three-trial conditioning. In all cases, control bees (Oi) learned significantly better than exposed bees (IPA). Thus, IPA exposure impairs subsequent associative olfactory learning irrespective of the odorant selected for conditioning. ${ }^{*} p<0.05$ (as compared to respective Oil control groups). 


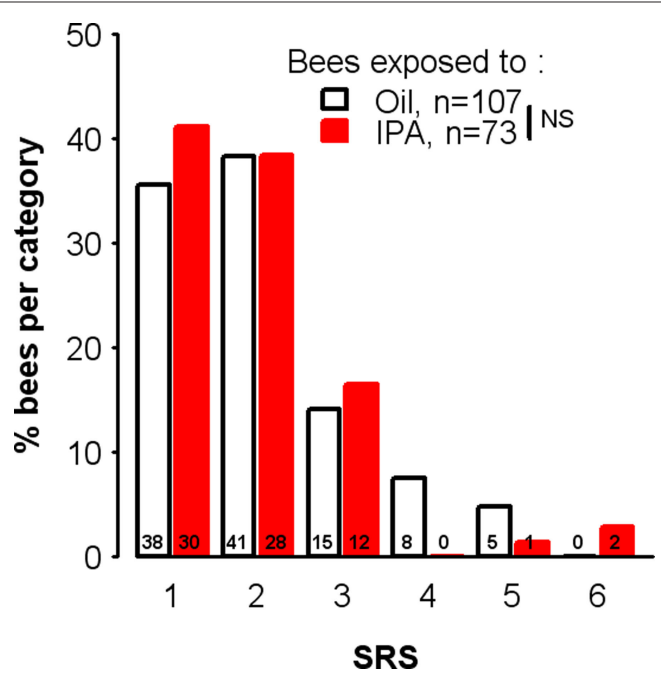

FIGURE 7 | Learning impairment after IPA exposure is not due to decreased sucrose responsiveness. Distribution of bees exposed to isopentyl acetate (IPA) or mineral oil (Oi) according to SRS (sucrose response score) values. Unconditioned responses elicited by sucrose were assessed, using 6 sucrose solutions of increasing concentrations $(0.1,0.3,1,3,10$, and $30 \%$, in water). For each animal, the presence or absence of a PER was recorded for each concentration, and its individual SRS was calculated as the number of stimuli eliciting a PER (e.g., SRS $=3$ for an individual responding to 3,10 , and $30 \%$ sucrose but not to lower concentrations). The number of bees with a given value in each group is indicated by the number on each bar. Among bees showing motivation to respond to sucrose, no difference was found between the two groups $\left(\chi^{2}=3.68, \mathrm{df}=3, p=0.30\right.$; bees with SRS values higher than three were grouped in a single category to allow the use of the chi-square test, see Materials and Methods). NS: non-significant.

2004; Rueppell et al., 2006; Roussel et al., 2009). We used this method to determine whether IPA-exposed and control bees differed in their sucrose sensitivity, independently of conditioning since we aimed to study the unconditioned response without prior experience of any sucrose reward. Bees were grouped in classes corresponding to their SRSs, ranging from 1 (low responsiveness, i.e., bees responding only to the highest sucrose concentration) to 6 (high responsiveness, i.e., bees responding to all six sucrose concentrations). Bees with a SRS of 0 , i.e., not responding to any sucrose concentration, were discarded to focus the analysis on bees showing appetitive motivation as in the learning experiments.

The distribution of IPA-treated and control bees among the different SRS classes is shown in Figure 7. We found no difference between IPA-exposed and control bees in terms of their SRS $\left(\chi^{2}=3.68, \mathrm{df}=3, p=0.30\right)$. Thus, among bees showing motivation to respond to sucrose (i.e., those whose learning performance was analyzed), exposure to IPA impaired learning without affecting responsiveness to the US.

\section{EXPOSURE TO IPA AFFECTS OLFACTORY GENERALIZATION}

In order to ask whether IPA might affect olfactory processing, we inverted the sequence of treatments: we conditioned first the bees with 1-nonanol as the CS during three conditioning trials and, immediately after the last conditioning trial, we exposed them to IPA, geraniol, or mineral oil. After the 30-min resting period following exposure, we performed an olfactory generalization test in which bees were presented with the CS (1-nonanol) and with two novel odorants, nonanal and 1-hexanol. While 1-nonanol and nonanal are perceived by bees as similar, 1-nonanol and 1-hexanol, are perceived as dissimilar (Guerrieri et al., 2005). As expected since bees were not exposed to any substance before conditioning, acquisition rates were equivalent in all three groups (two-way ANOVA, Trial: $F=227.2, \mathrm{df}=2,350, p<0.001$, Group: $F=0.26$, $\mathrm{df}=2,175, p=0.77)$. After exposure either to mineral oil, geraniol, or IPA, responses to the three odorants, 1-nonanol, nonanal, and 1-hexanol, differed between groups. While response levels to the CS were similar between groups $\left(\chi^{2}=1.40, \mathrm{df}=2, p=0.49\right)$, bees exposed to IPA displayed slightly but significantly lower levels of generalization (dissimilar odorant: $\chi^{2}=5.11, \mathrm{df}=2, p=0.08$, similar odorant: $\chi^{2}=10.1, \mathrm{df}=2, p=0.006$ ) (Figure 8A). In order to confirm this observation, we examined individual response profiles in the three groups. Bees were categorized as bees responding to the CS only, bees showing generalization toward the similar odorant, bees showing generalization to both the similar and dissimilar odorants, and bees responding to neither of the stimuli (Figure 8B). Overall, exposure significantly affected individual responses $\left(\chi^{2}=15, \mathrm{df}=6, p=0.02\right)$, with the most profound effect observed for specific responses (CS only): more than half (55\%) of the bees in this category were IPA-exposed bees. Conversely, IPA-exposed bees showed full generalization (response to the three odorants) twice as less often (21\%) as bees exposed to geraniol or oil (resp. 41 and 39\%). These results exclude the possibility that IPA exposure impairs odorant perception and discrimination, because the consequences of such exposure were opposite to those that one would predict under such hypothesis: instead of becoming less responsive or less selective to the conditioned odorant, IPA-exposed bees remained responsive to that odorant but increased their response selectivity, thus decreasing odorant generalization.

\section{DISCUSSION}

Learning and memory performances rely on a variety of intrinsic (related to the animal's internal state or physiology) and environmental factors. Studies on the behavioral and neural bases of learning and memory tend to focus on the former but less on the latter as environmental factors are usually viewed as external to the biological machinery mediating individual plasticity. However, environmental factors and among them, social ones, may dramatically influence physiological processes, and therefore the nervous system responsible for learning and memory processes. In the honeybee, much attention has been given to the modulation of learning and/or memory by intrinsic factors. Using the protocol of PER conditioning, learning has been shown to depend on age (Ray and Ferneyhough, 1999; Behrends et al., 2007), caste (Sigg et al., 1997; Behrends et al., 2007) and motivational state (Scheiner et al., 2001a,b). However, surprisingly in the case of a highly social insect, only a few studies have shown influences of the environment on plastic processes (Farina et al., 2005; Arenas et al., 2009), and particularly of social signals from conspecifics (Vergoz et al., 2007a). Pheromones, the main class of intraspecific communication signals in social insects, have been known for years to modulate reflex responses to a variety of stimuli, but it was only recently that effects on learning were reported. The QMP could impair aversive 


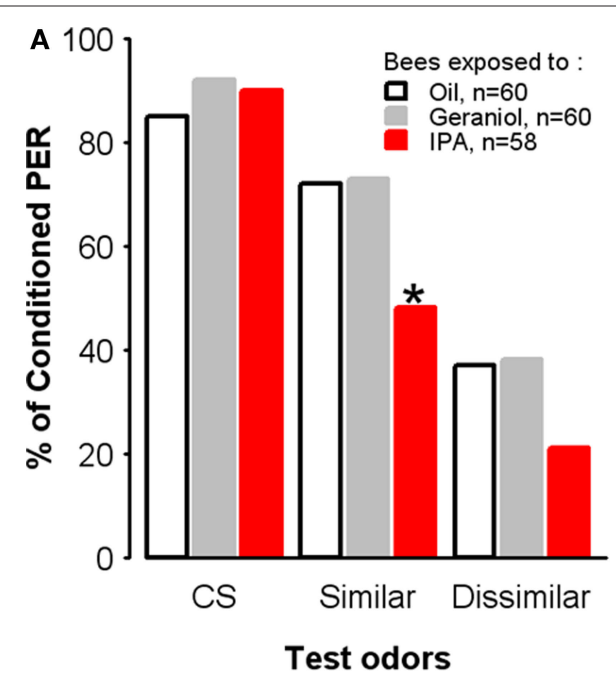

FIGURE 8 | Exposure to IPA affects olfactory generalization. Learning performances, as indicated by percentages of conditioned PER to the conditioned odor on the third conditioning trial, for each group. Independent groups of bees were exposed to oil, geraniol, or IPA, immediately after conditioning, and then tested $1 \mathrm{~h}$ after the end of conditioning. Generalization was assessed by measuring PER in response to unrewarded presentations of 1-nonanol (CS), nonanal (an odorant perceptually similar to 1-nonanol) and

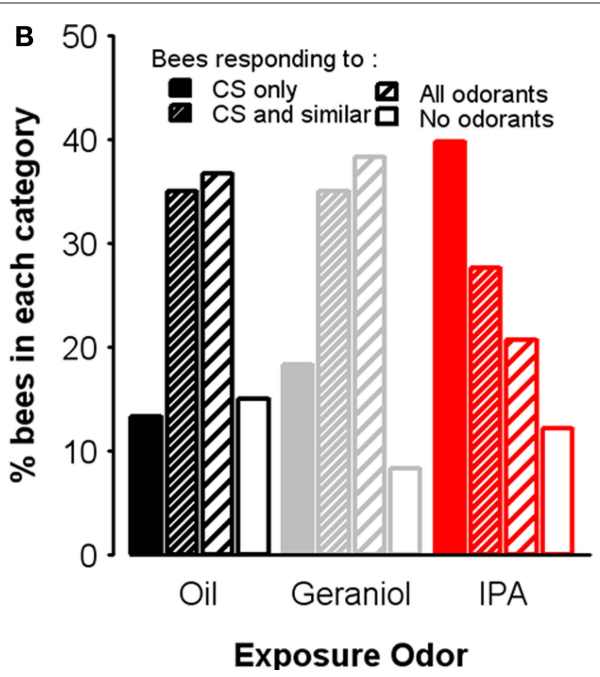

1-hexanol (perceived as dissimilar to 1-nonanol). (A) Percentages of conditioned responses to the three odorants, for each group. Bees exposed to IPA respond less to the similar odor. ${ }^{*} p<0.05$ (as compared with respective control groups). (B) Distribution of bees according to their individual response profiles. Specific responses to the CS are more frequent in bees exposed to IPA, while most control bees (Oil and Geraniol) show generalization responses. learning (olfactory conditioning of the sting extension reflex, SER), but not appetitive learning (olfactory conditioning of PER) (Vergoz et al., 2007a). We thus wondered whether other pheromones might modulate appetitive learning. Our results are the first experimental evidence of a pheromonal modulation of appetitive learning.

The SAP triggers aggressive behavior in worker honeybees and decreases the probability to engage into foraging for food sources (Free, 1987; Hunt, 2007; Nieh, 2010). From this point of view, the negative modulation of appetitive learning by SAP and by its main component, IPA, when used at ecologically relevant amounts, makes sense. As appetitive learning is one of the main processes mediating foraging activities of honeybees (Giurfa, 2007), depressing foraging activities, and concomitantly appetitive learning, might be part of a strategy that helps attending better potential aversive signals in a defensive context signalized by SAP and/or IPA. In such a context, discriminating "friend from foe" is important for efficient defense of the colony (Breed, 1983); it relies on olfactory cues such as cuticular hydrocarbons (e.g., Breed and Stiller, 1992; Châline et al., 2005; Dani et al., 2005), comb wax components (D'Ettorre et al., 2006) or chemicals contained in the vertebrates' breath (Breed et al., 2004). Considering the higher sensitivity to SAP of young guards compared with older foraging bees (see Hunt, 2007) and the clear age-dependence for the effect of QMP on aversive learning (Vergoz et al., 2007b), age and/or caste may be a critical factor for the modulation of learning by IPA. Since we did not consider age differences here, this crucial point deserves further examination.

As SAP is known as a releaser pheromone, its effects (or those of IPA) have been studied on a short timescale (over minutes). Interestingly, here the learning impairment after a 30-min exposure lasted up to $24 \mathrm{~h}$ (but not longer), which suggests that at least some effects of releaser pheromones may last longer than usually considered. Changes in learning performances over hours may involve plastic changes in the brains of IPA-exposed bees, such as modifications of synaptic transmission and/or neural excitability in the olfactory pathway. In this respect, different brain neuropiles involved in learning might be affected over different time-courses. Consistent with this idea, exposure to IPA was shown to affect the expression of immediate-early genes in the antennal lobes (the primary olfactory centers) (Alaux and Robinson, 2007). However, exposure to a plant odorant (hexanal) yielded the same result in that same study, so that it cannot be attributed exclusively to IPA. This unspecific action may be due to the very short exposure time (1 min) used in that work (Alaux and Robinson, 2007). Here, using a longer exposure time, we verified that exposure to another plant odorant (methyl salicylate) or to another pheromonal compound (geraniol) does not affect olfactory learning, so that the impairment induced by IPA in our work is indeed specific. As geraniol is a main element of the attractive Nasonov pheromone (Boch et al., 1962; Free, 1987), we interpret the effect of IPA or SAP as a learning impairment related to their biological significance as alarm signals and potential stress factors. It would be interesting to test for a specific up-regulation of immediate-early genes in our experimental conditions, which could lead to altered learning performances over a period of hours. Consistent with the idea of a general learning impairment, this effect could be reproduced using different odorants as CSs. Interestingly, appetitive learning is also impaired in bees exposed to physiological stress, either immune (Mallon et al., 2003; Iqbal and Mueller, 2007) or metabolic (Farooqui, 2008; Amdam et al., 2010). Thus, impaired learning may be part of a general response to stress in the honeybee. It may simply reflect the unavailability of cognitive resources aimed at 
solving an inappropriate problem (here appetitive learning) in a situation in which such resources should support responses in an aversive, defensive context.

Importantly, this effect cannot be interpreted as a mere consequence of impaired perception of the conditioned odorant (the CS), as bees exposed to IPA still discriminate the CS from a novel odorant in a generalization test (they do so even better than controls). Such a lower generalization combined with lower acquisition has been reported previously in aging forager bees, and interpreted as the result of a possible compensatory mechanism (Behrends et al., 2007). In our experimental context, an interesting hypothesis would be that SAP, or IPA, affects generalization levels by priming the olfactory system to focus specifically on signals that may be relevant in an aversive context indicated by the alarm pheromone. By contrast, exposure to an attractive pheromone like the Nasonov pheromone (or its main component, geraniol) did not alter generalization toward novel odorants. These results raise the interesting question of whether generalization differs between an aversive and an appetitive framework, irrespective of pheromonal exposure.

The decrease in learning performance was neither due to a decreased sensitivity to sucrose (the US). In a previous work, it was shown that bees exposed to IPA tend to respond less to sucrose (Balderrama et al., 2002). Our finding that more bees failed to respond to sucrose during conditioning after exposure to IPA confirms this result. However, in order to study the effects of IPA exposure on associative learning irrespective of a decrease in appetitive motivation, we discarded IPA-exposed bees that did not respond to the US. We thus kept bees showing consistent appetitive unconditioned responses, which enabled us to show that even in the case of an unaffected reward evaluation (as indicated by similar SRS values) acquisition is altered after exposure to IPA. It is clear, therefore, that the impairment of learning induced by IPA cannot be solely attributed to a deficit in reward perception or evaluation. All in all, the fact that processing of neither the CS nor the US was deteriorated in the bees used in our experiment suggests that exposure to IPA affects the ability to form the CS-US association itself, independently of effects on CS and/or US perception.

What could be the neurobiological substrate of such a response? If CS signaling is unaffected (and CS responses are even improved), and if US processing was unaffected in the bees selected for the experiments, how could IPA act on the association between CS and US in order to impair learning? Biogenic amines play key roles in the regulation of learning in insects (Giurfa, 2006), and QMP was shown to impair aversive learning by inhibiting dopamine signaling (Vergoz et al., 2007a). While dopamine has been mainly involved in aversive conditioning in insects, octopamine is crucial for appetitive learning (Schwaerzel et al., 2003; Riemensperger et al., 2005;

\section{REFERENCES}

Alaux, C., and Robinson, G. E. (2007). Alarm pheromone induces immediate-early gene expression and slow behavioral response in honey bees. J. Chem. Ecol. 33, 1346-1350.

Amdam, G. V., Fennern, E., Baker, N., and Rascon, B. (2010). Honeybee associative learning performance and metabolic stress resilience are positively associated. PLOS ONE 5, e9740. doi:10.1371/journal. pone.0009740.

Arenas, A., Giurfa, M., Farina, W. M., and Sandoz, J. C. (2009). Early olfactory experience modifies neural activity in the antennal lobe of a social insect at the adult stage. Eur. J. Neurosci. 30, 1498-1508.

Balderrama, N., Núñez, J., Guerrieri, F., and Giurfa, M. (2002). Different

Unoki et al., 2005; Vergoz et al., 2007b). In honeybees, both sucrose responsiveness and PER acquisition performance are enhanced (resp. decreased) after activation (resp. inhibition) of octopaminergic transmission (Hammer and Menzel, 1995; Menzel et al., 1999; Scheiner et al., 2002; Pankiw and Page, 2003). Consistent with this, levels of octopamine (and dopamine) are depressed in the brains of bees submitted to stressful treatments (Chen et al., 2008), as it has been proposed in other species (Chentsova et al., 2002). Still, other evidence shows that stress and/or pain can increase octopamine titers (Harris and Woodring, 1992; Möbius and Penzlin, 1993; Hirashima et al., 2007). These discrepancies may be linked to spatial (e.g., brain or hemolymph) and temporal variations in octopamine release. In any case, disruption of octopaminergic transmission is unlikely to be a unique and straightforward explanation for the effects of SAP exposure, as in the case of the response to oxidative stress (Farooqui, 2008). Indeed, the dissociation shown here between the impact of IPA exposure on sucrose responsiveness and learning supports this view. Dopamine might also be involved as it does not regulate exclusively aversive learning (Kim et al., 2007; Selcho et al., 2009). Considering the stable effect of exposure on learning observed here, expression changes in biogenic amine receptors in different brain neuropiles might also be involved, as dopamine receptors have been shown to be regulated after only 2 days of exposure to QMP (Beggs et al., 2007). Another possibility that deserves consideration is the fact that IPA exposure seems to activate the equivalent of an opioid system in honeybees (Balderrama et al., 2002). It has been suggested that such activation induces an analgesia-like state in honeybees, thus enhancing tolerance to potential noxious stimuli (Balderrama et al., 2002). Although this possibility seems interesting to consider, it is worth underlining that our bees are confronted with appetitive rather than with aversive conditioning after IPA exposure. An analgesia-like state, if any, would not be relevant in the case of a sucrose reinforcement experienced during olfactory learning. However, an alternative interpretation would posit that activation of a putative opioid-like system would be accompanied by learning impairment in any conditioning protocol, irrespective of its appetitive or aversive nature. Further experiments should help to understand the physiological bases of the neuromodulation of learning under stressful procedures.

\section{ACKNOWLEDGMENTS}

This study was supported by a grant of the University Paul Sabatier (APIGENE), a PEPS grant from the CNRS to Jean-Marc Devaud. and a Gateway Grant from the Research Center on Animal Cognition to Jean-Marc Devaud and Bernard Francés. The authors wish to thank Simon Lepicard for his help on preliminary experiments, and Sandrine Paute for providing the bees necessary for the experiments.

functions of two alarm substances in the honeybee. J. Comp. Physiol. A Neuroethol. Sens. Neural. Behav. Physiol. 188, 485-491.

Beggs, K. T., Glendining, K. A., Marechal, N. M., Vergoz, V., Nakamura, I., Slessor, K. N., and Mercer, A. R. (2007). Queen pheromonemodulates brain dopamine function in worker honey bees. Proc. Natl. Acad. Sci. U.S.A. 104, 2460-2464.
Behrends, A., Scheiner, R., Baker, N., and Amdam, G. V. (2007). Cognitive aging is linked to social role in honey bees (Apis mellifera). Exp. Gerontol. 42, 1146-1153.

Ben-Shahar, Y. (2005). The foraging gene, behavioral plasticity, and honeybee division of labor. J. Comp. Physiol. A 191, 987-994.

Bitterman, M. E., Menzel, R., Fietz, A., and Schafer, S. (1983). Classical 
conditioning of proboscis extension in honeybees (Apis mellifera). J. Comp. Psychol. 97, 107-119.

Boch, R., and Shearer, D. A. (1962). Identification of geraniol as the active component in the nassanoff pheromone of the honey bee. Nature 194, 704-706.

Boch, R., Shearer, D. A., and Stone, B. C. (1962). Identification of isoamyl acetate as an active component in the sting pheromone of the honey bee. Nature 195, 1018-1020.

Breed, M. D. (1983). Nestmate recognition in honey bees. Anim. Behav. 31, 86-91.

Breed, M. D., Guzmán-Novoa, E., and Hunt, G. J. (2004). Defensive behavior of honey bees: organization, genetics, and comparisons with other bees. Annu. Rev. Entomol. 49, 271-298.

Breed, M. D., and Stiller, T. M. (1992). Honey bee, Apis mellifera, nestmate discrimination- hydrocarbon effects and the evolutionary implications of comb choice. Anim. Behav. 43, 875-883

Châline, N, Sandoz, J. C., Martin, S. J., Ratnieks, F. L. W., and Jones, G. R. (2005). Learning and discrimination of individual cuticular hydrocarbons by honeybees (Apis mellifera). Chem. Senses 30, 327-335.

Chen, Y. L., Hung, Y. S., and Yang, E. C. (2008). Biogenic amine levels change in the brains of stressed honeybees. Arch. Insect Biochem. Physiol. 68, 241-250.

Chentsova, N. A., Gruntenko,N. E., Bogomolova, E. V., Adonyeva, N. V., Karpova, E. K., and Rauschenbac, I. Y. U. (2002). Stress response in Drosophila melanogaster strain inactive with decreased tyramine and octopamine contents. J. Comp. Physiol. B, Biochem. Syst. Environ. Physiol. 172, 643-650

Collins, A. M., and Blum, M. S. (1983). Alarm responses caused by newly identified compounds derived from the honeybee sting. J. Chem. Ecol. 9 , 57-65.

Dani, F. R., Jones, G. R., Corsi, S., Beard, R., Pradella, D., and Turillazzi, S. (2005). Nestmate recognition cues in the honey bee: differential importance of cuticular alkanes and alkenes. Chem. Senses 30, 477-489.

D’Ettorre, P., Wenseleers, T., Dawson, J., Hutchinson, S., Boswell, T., and Ratnieks, F. L. W. (2006). Wax combs mediate nestmate recognition by guard honeybees. Anim. Behav. 71, 773-779.

Farina, W. M., Gruter, C., and Diaz, P. C. (2005). Social learning of floral odours inside the honeybee hive. Proc. Biol. Sci. 272, 1923-1928.

Farooqui, T. (2008). Iron-induced oxidative stress modulates olfactory learn- ing and memory in honeybees. Behav. Neurosci. 122, 433-447.

Free, J. B. (1987). Pheromones of Social Bees. Ithaca: Comstock Publishing Associates.

Frisch, K. v. (1967). The Dance Language and Orientation of Bees. Cambridge, MA: Harvard University Press.

Giurfa, M. (2006). Associative learning: the instructive function of biogenic amines. Curr. Biol. 16, R892-5.

Giurfa, M. (2007). Behavioral and neural analysis of associative learning in the honeybee: a taste from the magic well. J. Comp. Physiol. A Neuroethol. Sens. Neural. Behav. Physiol. 193, 801-824.

Guerrieri, F., Schubert, M., Sandoz, J. C., and Giurfa, M. (2005). Perceptual and neural olfactory similarity in honeybees. PLoS Biol. 3, e60. doi: 10.1371/ journal.pbio.0030060.

Hammer, M., and Menzel, R. (1995). Learning and memory in the honeybee. J. Neurosci. 15, 1617-1630.

Harris, J. W., and Woodring, J. (1992). Effects of stress, age, season, and source colony on levels of octopamine, dopamine and serotonin in the honey bee (Apis mellifera, L.) brain. J. Insect. Physiol. 38, 29-35.

Hirashima, A., Yamaji, H., Yoshizawa, T., Kuwano, E., and Eno, M. (2007). Effect of tyramine and stress on sexpheromone production in the preand post-matink silkworm moth, Bombyx mori. J. Insect Physiol. 53, 1242-1249.

Hunt, G. J. (2007). Flight and fight: a comparative view of the neurophysiology and genetics of honey bee defensive behavior. J. Insect Physiol. 53, 399-410.

Hunt, G. J., Wood, K. V., Guzman-Novoa, E., Lee, H. D., Rothwell, A. P., and Bonham, C. C. (2003). Discovery of 3-methyl-2-buten-1-yl acetate, a new alarm component in the sting apparatus of Africanized honeybees. J. Chem. Ecol. 29, 453-463.

Iqbal, J., and Mueller, U. (2007). Virus infection causes specific learning deficits in honeybee foragers. Proc. Biol. Sci. 274, 1517-1521.

Kim, Y. C., Lee, H. G., and Han, K. A. (2007). D1 dopamine receptor dDA1 is required in the mushroom body neurons for aversive and appetitive learning in Drosophila. J. Neurosci. 27, 7640-7647.

Lunney, G. H. (1970). Using analysis of variance with a dichotomous dependent variable: an empirical study. J. Educat. Meas. 7, 263-269.

Mallon, E. B., Brockmann,A., and SchmidHempel, P. (2003). Immune response inhibits associative learning in insects. Proc. Biol. Sci. 270, 2471-2473.

Menzel, R. (1985). "Experimental behavioral ecology and sociobiology," in
Memoriam Karl von Frisch, 1886-1982, eds B. Holldobler and M. Lindauer (Stuttgart/New York: Gustav Fisher Verlag). 55-74.

Menzel, R.,Heyne,A., Kinzel, C., Gerber, B. and Fiala, A. (1999). Pharmacological dissociation between the reinforcing, sensitizing, and response-releasing functions of reward in honeybee classical conditioning. Behav. Neurosci. 113, 744-754.

Möbius, P., and Penzlin, H. (1993). Stressinduced release of octopamine in the American cockroach Periplaneta americana. Acta. Biol. Hung. 44 45-50.

Nieh, J. C. (2010). A negative feedback signal that is triggered by peril curbs honey bee recruitment. Curr. Biol. 20, 310-315.

Núñez, J., Almeida, L., Balderrama, N., and Giurfa, M. (1998). Alarm pheromone induces stress analgesia via an opioid system in the honeybee. Physiol. Behav. 63, 75-80.

Page, R. E. Jr., and Erber, J. (2002). Levels of behavioral organization and the evolution of division of labor Naturwissenschaften 89, 91-106.

Page, R. E. Jr., Scheiner, R., Erber, J., and Amdam, G. V. (2006). The development and evolution of division of labor and foraging specialization in a social insect (Apis mellifera L.). Curr. Top. Dev. Biol. 74, 253-286.

Pankiw, T. (2003). Directional change in a suite of foraging behaviors in tropical and temperate evolved honey bees (Apis mellifera L.). Behav. Ecol. Sociobiol. 54, 458-464.

Pankiw, T. (2005). "The honey bee foraging behavior syndrome: quantifying the response threshold model of division of labor," in Proceedings of the 2005 IEEE Swarm Intelligence Symposium, eds P. Arabshahi and A. Martinoli (Pasadera: IEE), 1-6.

Pankiw, T., and Page, R. E. Jr. (2003) Effect of pheromones, hormones, and handling on sucrose response thresholds of honey bees (Apis mellifera L.). J. Comp. Physiol. A Neuroethol. Sens. Neural. Behav. Physiol. 189, 675-684.

Ray, S., and Ferneyhough, B. (1999). Behavioral development and olfactory learning in the honeybee (Apis mellifera). Dev. Psychobiol. 34 21-27.

R Development Core Team (2009). R: a language and environment for statistical computing. R Foundation for Statistical Computing, Vienna, Austria URL http://www.R-project.org

Riemensperger, T., Voller, T., Stock, P. Buchner, E., and Fiala, A. (2005). Punishment prediction by dopaminergic neurons in Drosophila. Curr. Biol. 15, 1953-1960.
Robinson, G., and Page, R. (1989). "Genetic basis for division of labor in an insect society," in The Genetics of Social Evolution, eds M. Breed and R. Page (Boulder, CO: Westview Press Inc), 61-82.

Roussel, E., Carcaud, J., Sandoz, J. C., and Giurfa, M. (2009). Reappraising social insect behavior through aversive responsiveness and learning. PLoS ONE 4, e4197. doi: 10.1371/journal. pone.0004197.

Rueppell, O., Chandra, S. B., Pankiw, T., Fondrk, M. K., Beye, M., Hunt, G., and Page, R. E. (2006). The genetic architecture of sucrose responsiveness in the honeybee (Apis mellifera L.). Genetics 172, 243-251.

Scheiner, R., Erber, J., and Page, R. E. Jr. (1999). Tactile learning and the individual evaluation of the reward in honey bees (Apis mellifera L.). J. Comp. Physiol. A185, 1-10.

Scheiner, R., Page, R. E. Jr., and Erber, J. (2001a). The effects of genotype, foraging role, and sucrose responsiveness on the tactile learning performance of honey bees (Apis mellifera L.). Neurobiol. Learn. Mem. 76, 138-150.

Scheiner, R., Page, R. E. Jr., and Erber, J. (2001b). Responsiveness to sucrose affects tactile and olfactory learning in preforaging honey bees of two genetic strains. Behav. Brain Res. 120, 67-73.

Scheiner, R., Page, R. E., and Erber, J. (2004). Sucrose responsiveness and behavioral plasticity in honeybees (Apis mellifera). Apidologie 35, 133-142.

Scheiner, R., Pluckhahn, S., Oney, B., Blenau, W., and Erber, J. (2002). Behavioural pharmacology of octopamine, tyramine and dopamine in honey bees. Behav. Brain Res. 136, 545-553.

Schwaerzel, M., Monastirioti, M., Scholz H., Friggi-Grelin, F., Birman, S., and Heisenberg, M. (2003). Dopamine and octopamine differentiate between aversive and appetitive olfactory memories in Drosophila. J. Neurosci. 23, 10495-10502.

Selcho, M., Pauls, D., Han, K. A., Stocker, R. F., and Thum, A. S. (2009). The role of dopamine in Drosophila larval classical olfactory conditioning. PLoS ONE 4, e5897. doi:10.1371/journal. pone.0005897.

Sigg, D., Thompson, C. M., and Mercer,A. R. (1997).Activity-dependent changes to the brain and behavior of the honey bee, Apis mellifera (L.). J. Neurosci. 17, 7148-7156.

Takeda, K. (1961). Classical conditioned response in the honeybee. J. Insect Physiol. 6, 168-179. 
Unoki, S., Matsumoto, Y., and Mizunami, M. (2005).Participation of octopaminergic reward system and dopaminergic punishment system in insect olfactory learning revealed by pharmacological study. Eur. J. Neurosci. 22, 1409-1416.

Vander Meer, R. K., Breed, M. D., Winston, M., and Espelie, K. E. (1997). Pheromone Communication In Social Insects: Ants, Wasps, Bees, And Termites. Boulder: Westview Press.

Vergoz, V., Schreurs, H. A., and Mercer, A. R. (2007a). Queen pheromone blocks aversive learning in young worker bees. Science 317, 384-386.

Vergoz, V., Roussel, E., Sandoz, J. C., and Giurfa, M. (2007b). Aversive learning in honeybees revealed by the olfactory conditioning of the sting extension reflex. PLOS ONE2, e288. doi:10.1371/ journal.pone.0000288.

Wilson, E. O. (1971). The Insect Societies. Harvard: Belknap Press of Harvard University Press.

Wilson, E. O., and Hölldobler, B. (2009). The Superorganism: The Beauty, Elegance, and Strangeness of Insect
Societies. New York, NY: WW Norton \& Co.

Conflict of Interest Statement: The authors declare that the research was conducted in the absence of any commercial or financial relationships that could be construed as a potential conflict of interest.

Received: 12 July 2010; paper pending published: 23 July 2010; accepted: 08 August 2010; published online: 30 August 2010.
Citation: Urlacher E, Francés B, Giurfa $M$ and Devaud J-M (2010) An alarm pheromone modulates appetitive olfactory learning in the honeybee (Apis mellifera). Front. Behav. Neurosci. 4:157. doi: 10.3389/fnbeh.2010.00157

Copyright (C) 2010 Urlacher, Francés, Giurfa and Devaud. This is an open-access article subject to an exclusive license agreement between the authors and the Frontiers Research Foundation, which permits unrestricted use, distribution, and reproduction in any medium, provided the original authors and source are credited. 